\title{
评述 分子荧光探针专刊
}

\section{$\beta$-半乳糖苷酶苂光探针的研究进展}

\author{
李檬 ${ }^{1}, \operatorname{la}^{\text {天 }}$ 初 $^{2}$, 朱为宏 ${ }^{3 *}$
}

1. 华北电力大学环境科学与工程系, 保定 071000

2. 保定市第一中心医院, 保定 071000

3. 华东理工大学化学与分子工程学院, 教育部结构可控先进功能材料及其制备重点实验室与精细化工研究所, 上海市功能材料化学重点 实验室, 上海 200237

*通讯作者, E-mail: whzhu@ecust.edu.cn

收稿日期: 2017-02-25; 接受日期: 2017-04-17; 网络版发表日期: 2017-07-13

国家自然科学基金(编号: 21607044)、河北省自然科学基金(编号: B2017502069)和中央高校基本科研业务费专项资金(编号: 2016MS108) 资助项目

\begin{abstract}
摘要 $\beta$-半乳糖苷酶是细胞溶酶体中的水解酶, 其重要的生理功能是催化水解糖苷键, 将乳糖转化成半乳 糖. 在遗传学领域中对人类 $\beta$-半乳糖苷酶缺陷病的诊断和基础研究也是重要的指征, 故 $\beta$-半乳糖苷酶活性的 检测在生命医学领域具有重要意义. 近年来, 荧光探针因具有选择性好、灵敏度高及高时空分辨率成像等 优点, 深受研究者的青睐, 已发展了大量的可用于生物体系内 $\beta$-半乳糖苷酶识别与可视化成像的荧光探针. 本文对识别 $\beta$-半乳糖苷酶的荧光探针的设计策略以及研究进展进行了综述, 对未来 $\beta$-半乳糖苷酶识别荧光 探针的研究方向进行了展望.
\end{abstract}

关键词 $\beta$-半乳糖苷酶, 荧光, 探针, 细胞成像

\section{1 引言}

$\beta$-半乳糖苷酶( $\beta$-galactosidase, $\beta$-Gal, EC 3.2.1.23) 常简称为乳糖酶, 广泛存在于各种动物、植物及微生 物中, 是一种重要的具有生理病理作用的水解酶 ${ }^{[1 ~ 3]}$. 其主要生理功能是催化水解糖苷键, 将乳糖转化成 半乳糖, 在维持正常的生命活动中具有举足轻重的作 用. $\beta$-半乳糖苷酶活性及含量的异常通常与癌症的发 生有着密切的关系 ${ }^{[4,5]}$. 此外, 在遗传学领域中对人类 $\beta$-半乳糖苷酶缺陷病的诊断和基础研究也是重要的 指征 ${ }^{[6,7]}$. 研发高效的检测手段来实现对生物体系中 $\beta$ 半乳糖苷酶的活性检测和成像对深入研究重大疾病
的发病机制具有不可忽视的作用. 传统的酶活性分析 检测手段有比色法 ${ }^{[8]}$ 、电化学法 ${ }^{[9,10]}$ 等, 但因各自的局 限性都无法实现对生物体系内 $\beta$-半乳糖苷酶的含量 进行实时原位无损的检测. 近年来, 苂光探针法因其 具有选择性好、灵敏度高、操作简便, 可实现实时快 速、原位无损检测、高时空分辨率成像等优势 ${ }^{[11 ~ 15]}$, 已广泛用于活细胞水平离子及小分子的识别及细胞 成像 ${ }^{[16-20]}$, 同时也成为生物体系中 $\beta$-半乳糖苷酶活性 检测与可视化成像的一种重要手段, 成为医学诊断领 域的研究热点 ${ }^{[21 ~ 24]}$. 基于酶的催化专一性特点, $\beta$-半乳 糖苷酶可以特异性识别 $\beta$-半乳糖苷键. 因此, 对于 $\beta$-半 乳糖苷酶的检测常采用反应激活型苂光探针, 该类探

引用格式: 李檬, 高天初, 朱为宏. $\beta$-半乳糖苷酶苂光探针的研究进展. 中国科学: 化学, 2017, 47: 898-905 Li M, Gao T, Zhu W. Progress in fluorescent sensors for $\beta$-galactosidase. Sci Sin Chim, 2017, 47: 898-905, doi: 10.1360/N032017-00030 
针能够通过酶的高效催化转化能力实现苂光信号的 放大和高检测灵敏度 ${ }^{[25 ~ 27]}$. 本文针对 $\beta$-半乳糖苷酶的 反应激活型苂光探针的研究进展进行综述, 基于在细 胞成像以及活体应用方面, 阐述苂光增强型、比率型 菼光探针的设计策略以及细胞成像中的应用, 尤其是 双光子荧光探针的应用.

\section{2 苂光增强型 $\beta$-半乳糖苷酶探针}

\section{1 设计策略}

相对于苂光猝灭型探针, 苂光增强型探针具有干 扰相对较小、检测限相对较低、更适于生物体系等 优点, 因此得到了极大的关注 ${ }^{[28,29]}$. 针对 $\beta$-半乳糖苷酶 的检测, 该类型探针多在现有的苂光团的母体上引入 保护基团(如半乳糖), 在保护基团的保护下, 苂光团处 于苂光猝灭状态. 一旦与 $\beta$-半乳糖苷酶识别, 酶的催 化作用使保护基团裂解, 荧光团的苂光重新恢复, 从 而达到苂光响应的目的(图1).

\section{2 研究进展}

近年来, 研究者设计合成出了许多性能优异的苂 光增强型 $\beta$-半乳糖苷酶探针, 为 $\beta$-半乳糖苷酶荧光探 针的开发和理论研究奠定了基础. 十多年来, 日本东 京大学的Nagano团队设计合成了一系列 $\beta$-半乳糖苷 酶苂光探针, 取得了优异的成果.

2005 年, Nagano 等 ${ }^{[30]}$ 设计合成了一个以苂光素为 苂光基团的 $\beta$-半乳糖苷酶探针 1 (图2). 此探针在苂光 素的基础上, 对中位苯环 2 号位进行修饰将羧基替换 成甲基, 并将 4 号位引入新的基团甲氧基, 氧蒽杂环上 的酚羟基被半乳糖取代. 探针1采用光诱导电子转移 (photon-induced electron transfer, PET) 发光机理, 其自 身的荧光几乎处于猝灭状态. 当 $\beta$-半乳糖苷酶与探针

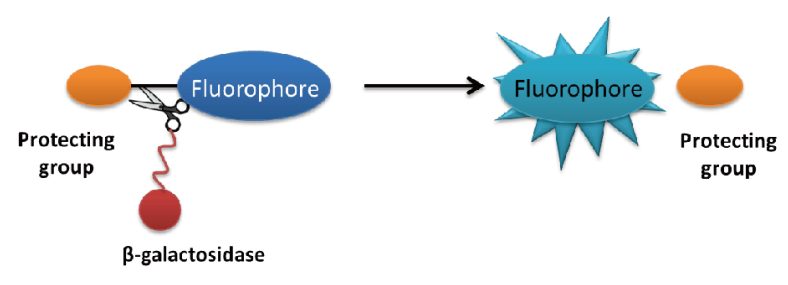

图 1 苂光增强型 $\beta$-半乳糖苷酶探针的设计策略(网络版 彩图)
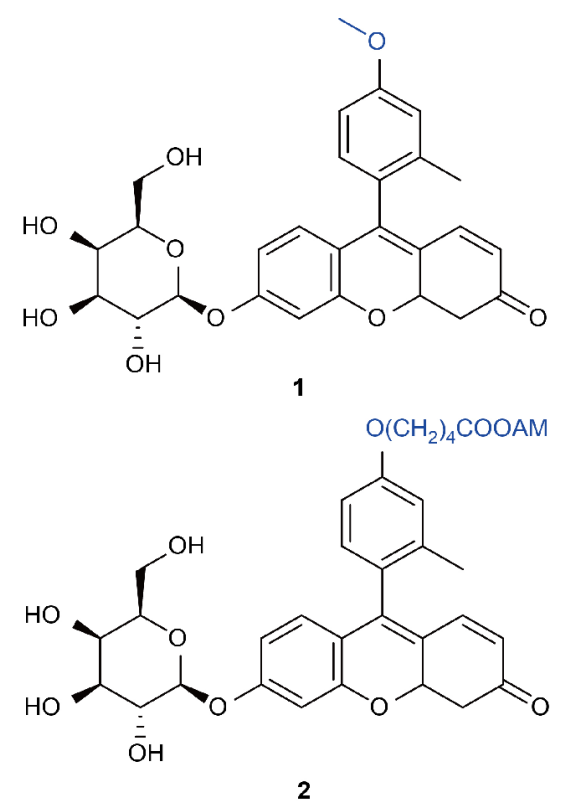

图 2 探针 1 和探针 $\mathbf{2}$ 的化学结构式 ${ }^{[30,31]}$ (网络版彩图)

识别后, 酶的催化作用使半乳糖从探针分子上断裂下 来, 所生成的产物在中性或碱性环境下以去质子化的 苯酚离子形式存在, 此时中位苯环部分对氧葱杂环的 PET效应大大减弱, 苂光发射得以恢复, 在 $509 \mathrm{~nm}$ 处可 得到 400 多倍的荧光增强响应, 但其细胞成像的效果 很不理想, 主要原因是水解后的产物在细胞中的保留 效果较差.

为了解决这一问题, Nagano等 ${ }^{[31]}$ 于 2007 年设计合 成了新型的苂光素探针 2 (图2). 该探针在探针 1 的甲 氧基位置引入乙酰羟甲基酯来提高其在细胞中的保 留能力. 探针进入细胞被 $\beta$-半乳糖苷酶水解后, 紧接 着被细胞内的酯酶水解, 使得探针以羧酸离子形式存 在, 亲水性大大增强, 不容易扩散出细胞, 从而实现细 胞内 $\beta$-半乳糖苷酶的高分辨率成像. 但是该探针也有 局限性, 只有在两种酶的协同作用下才能使苂光恢复, 响应时间较慢. 而且酯酶在细胞外区域普遍存在, 可 能会使得探针未进入细胞内之前就被酯酶水解成水 溶性的羧酸盐形式, 影响其进入细胞的能力, 使得活 体内成像效果降低.

为了解决上述问题, Nagano 等 ${ }^{[32]}$ 又于 2011 年报道 了一个新型的 $\beta$-半乳糖苷酶苂光探针 3 (图3). 该探针 不再采用PET发光机理, 而是以氧葱杂环类探针的螺 环开闭机理设计而成. 探针 $\mathbf{3}$ 自身在生理条件下以螺 环形式存在, 处于苂光猝灭状态. 经 $\beta$-半乳糖苷酶催化 

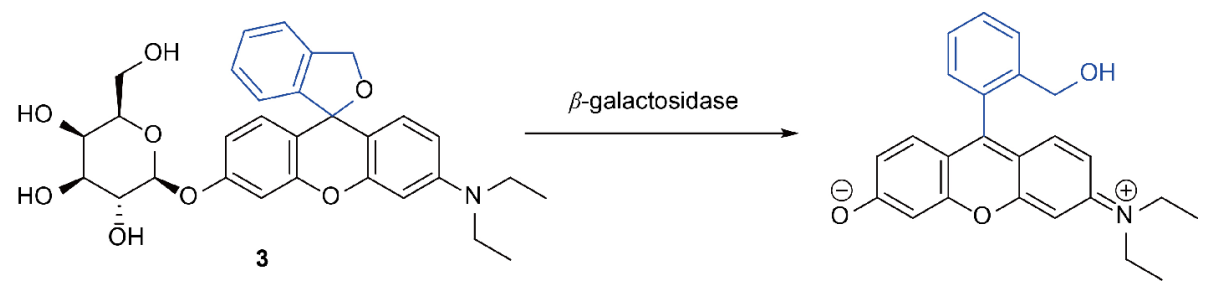

图 3 探针 3 与 $\beta$-半乳糖苷酶的响应机理 ${ }^{[32]}$ (网络版彩图)

水解后, 生成的产物以开环形式存在, 苂光显著增强. 将这一探针应用在细胞及果蝇组织中进行高分辨率 成像, 结果显示, 探针 $\mathbf{3}$ 可以很好地克服细胞穿透能力 限制, 并能保留在细胞内, 但该探针存在较高的荧光 背景强度, 并不能应用在小鼠肿瘤模型内 $\beta$-半乳糖苷 酶的成像.

2011年, Nagano等 ${ }^{[33]}$ 还报道了一系列苂光素染料, 为荧光素染料的设计合成提供了新的思路, 并且成功 地合成了苂光探针 4 用于 $\beta$-半乳糖苷酶的检测(图4). 该探针将氧杂葱环 10 位的氧原子用硅原子替代, 将吸 收和荧光光谱红移了 $90 \mathrm{~nm}$, 从而大大提高了探针的 信噪比. 因此, 该探针成功地应用于HEK293细胞中进 行红外区域的 $\beta$-半乳糖苷酶的检测, 并为活体成像的 发展奠定了基础.

为了提高 $\beta$-半乳糖苷酶苂光探针在活体成像中的 应用, Nagano等 ${ }^{[34]}$ 于 2015 年设计合成了又一新型的探 针 $\mathbf{5}$ (图5). 该探针在探针 $\mathbf{3}$ 的基础上, 引入了强吸电子
基团亚甲基三氟化碳结构单元, 从而提高闭环状态的 存在比例, 进而降低探针分子的背景荧光强度, 提高 了探针的信噪比, 故成功地实现小鼠肿瘤模型内 $\beta$-半 乳糖苷酶的识别及高分辨成像. 此外, 该团队还将探 针 5 用于 7 种卵巢癌细胞内 $\beta$-半乳糖苷酶的可视化苂 光成像, 表明该探针具有应用于临床上诊断卵巢癌转 移和荧光指导手术切除肿瘤的潜能.

除了以上对荧光素的研究和探索外, Nagano等还 对其他苂光团进行了研究. 2009年, Nagano等 ${ }^{[35]}$ 设计 合成了一例以噻唑橙为荧光团的探针 6 (图6), 该探针 通过单次甲基键将苯并噻唑和喹啉相连, 并对喹啉环 进行修饰得到自身荧光微弱的探针分子, 用于 $\beta$-半乳 糖苷酶的检测. 探针 $\mathbf{6}$ 是在苂光团上修饰了大体积亲 水的半乳糖结构, 此结构会阻断探针分子与生物分子, 尤其是DNA分子的结合, 从而使其被核膜隔开, 受染 色质蛋白的作用导致荧光发射减弱. 经 $\beta$-半乳糖酶水 解后, 半乳糖被去除, 从而使荧光团的苂光得以恢复.<smiles>Cc1ccccc1C1=C2C=CC(=O)C=C2[Si](C)(C)c2cc(OC3O[C@H](CO)[C@@H](O)[C@H](O)[C@H]3O)ccc21</smiles><smiles>Cc1ccccc1C1=C2C=CC(=O)C=C2[Si](C)(C)c2cc(Cl)ccc21</smiles>

图 4 探针 4 与 $\beta$-半乳糖苷酶的响应机理 ${ }^{[33]}$ (网络版彩图)

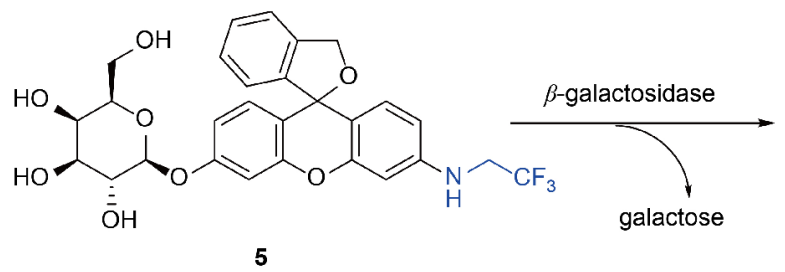<smiles></smiles>

图 5 探针 5 与 $\beta$-半乳糖苷酶的响应机理 ${ }^{[34]}$ (网络版彩图) 

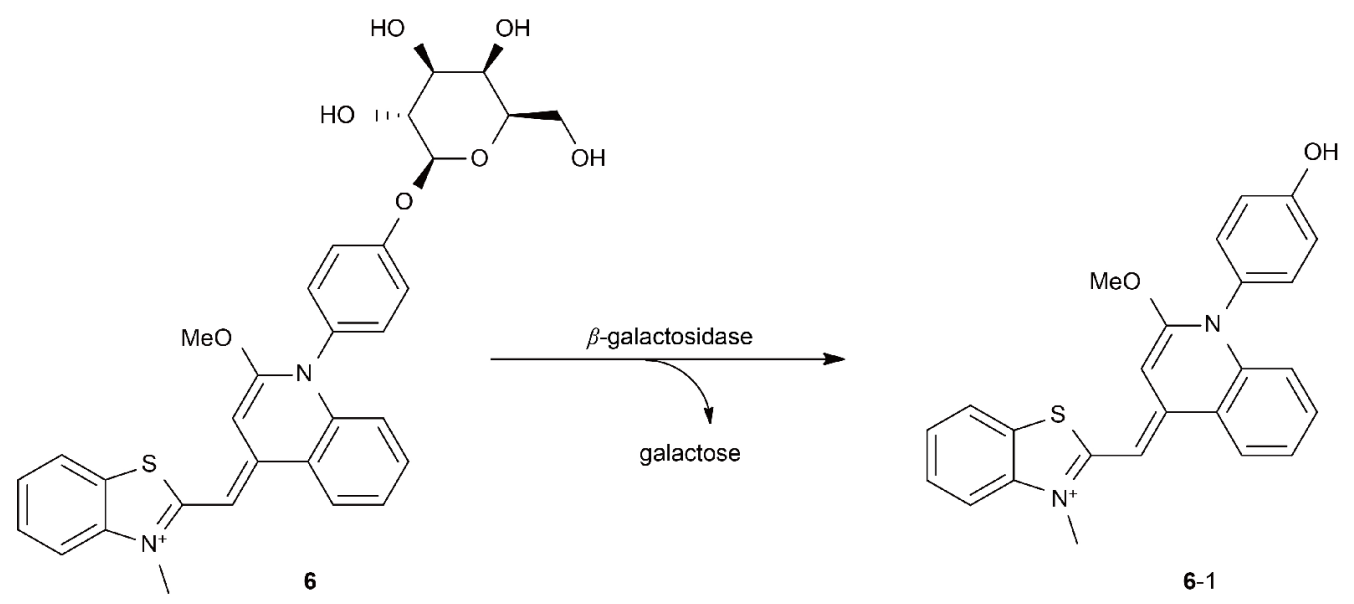

图 6 探针 6 与 $\beta$-半乳糖苷酶的响应机理 ${ }^{[35]}$

探针 6 及其水解产物 6-1, 分别应用在人宫颈癌细胞系 (HeLa) 中进行成像, 在光激发下探针 6 几乎没有荧光, 6-1有很强的绿色荧光, 但是 6-1 转移到细胞核内导致 了DNA断裂细胞死亡. 利用该特点, 进一步将探针 6 应 用在LacZ基因转染的HEK293细胞中, 通过与未转染 的细胞比较, 发现转染的细胞内有较强的荧光. 研究 表明, 探针 $\mathbf{6}$ 是一个良好的 $\beta$-半乳糖苷酶激发的光敏 剂, 对于光能疗法的发展起到了推动的作用.

除了日本东京大学的Nagano教授外, 世界各地的 科学家也为 $\beta$-半乳糖苷酶的苂光检测做出了不解的 努力, 取得了优异的成果. 2013年, Tung等 ${ }^{[36]}$ 报道了一 个以菁染料为荧光团的探针 7 , 并成功地将其应用于 C6/LacZ细胞成像中(图7). 研究显示, 探针7因带有未 水解的乙酰基, 可进入细胞. 经 $\beta$-半乳糖苷酶水解后 可得到一个近红外区域内的高荧光产物, 并可在细胞 内保留数天. 据推测探针 7 中的 $\alpha, \beta$ 不饱和亚胺离子在 细胞中受到含硫的亲核试剂进攻, 形成稀胺的中间体, 经质子化再次重排成亚胺离子, 在经过Knoevenagel缩 合反应和水解, 形成含有醛基的产物, 最后经过 $\beta$-半乳 糖苷酶的水解去除糖残基形成高苂光产物. 图7(b)为 特异性线粒体标记探针成像, 图7(f)为特异性内质网 标记探针成像, 图7(c, g) 为探针 7成像, 由图7(d, h) 的合 并成像图可以看出, 苂光信号仅存在于线粒体内, 该 探针是一个很好的线粒体标记物.

2014年, Shabat等 ${ }^{[37]}$ 设计合成了又一新型的菁染 料探针 8 (图8), 该探针在近红外染料上引入半乳糖, 使 得苂光团在 $720 \mathrm{~nm}$ 处几乎没有荧光, 在 $\beta$-半乳糖苷酶 的水解作用下, 探针释放出菁染料苂光团使得苂光得
(A)
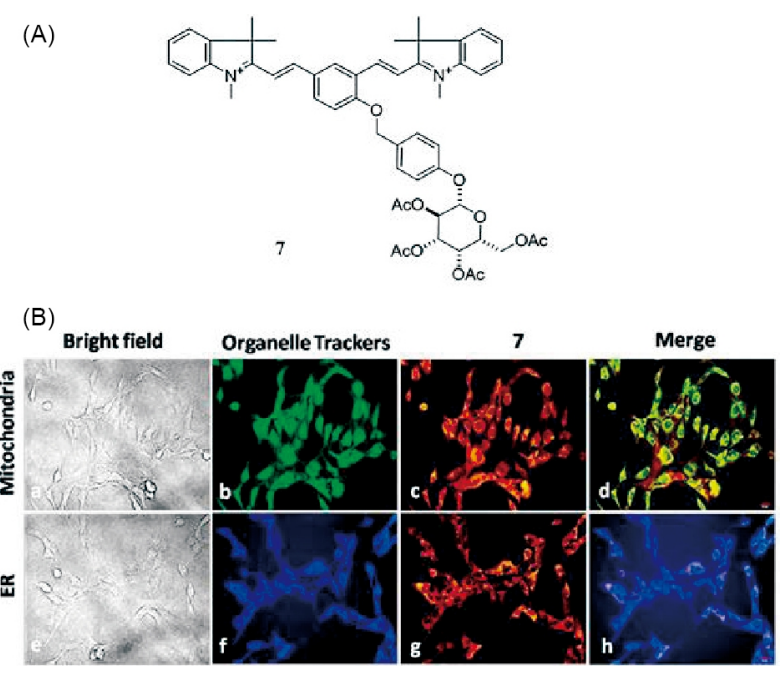

图 7 探针 7 的化学结构式(A)及特异性线粒体成像图(B). (a, e) 白光图像; (b) 线粒体绿色荧光标记的荧光图像; (f) 内 质网蓝色荧光标记的荧光图像; (c, g) 探针 7标记的苂光图 像; $(d, h)$ 合并后的苂光图像 ${ }^{[36]}$ (网络版彩图)

以恢复, 实现对 $\beta$-半乳糖苷酶高荧光比例检测, 并且 该探针成功地应用在小鼠模型内 $\beta$-半乳糖苷酶活性的 高分辨率三维成像. 此外, 该探针还为其他菁染料探 针的设计合成提供了新的模板.

\section{3 菼光比率型 $\beta$-半乳糖苷酶探针}

\section{1 设计策略}

与增强型荧光探针相比, 比率型荧光探针利用两 个波长处的苂光强度比例变化显示酶活性变化, 因此, 
(a)

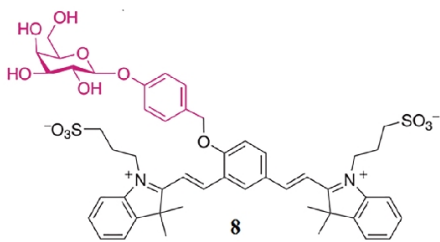

(b)

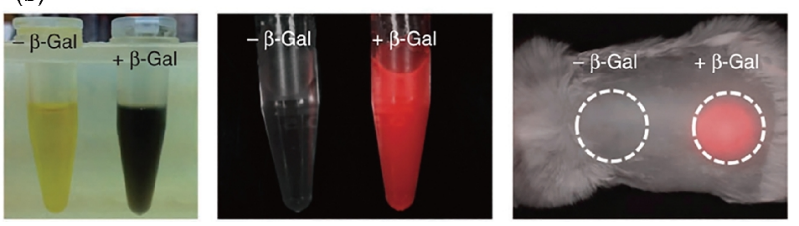

图 8 探针 8 的化学结构式(a)及其在小鼠体内的近红外苂 光成像图(b) ${ }^{[37]}$ (网络版彩图)

可以很好地排除探针浓度、测量环境因素、激发光 源强度、仪器测量误差等方面因素的影响, 实现高精 确度的检测, 尤其在生物体系中的检测方面具有独特 的优势 ${ }^{[38,39]}$.

此类型探针通常有两种设计策略. (1) 基于分子 内电荷转移 (intramolecular charge transfer, ICT) 效应, 利 用探针在与底物识别前后的荧光团的ICT效应的强弱 不同, 使得荧光发生红移或蓝移, 从而得到不同波长 处的荧光强度比例 ${ }^{[40,41]}$. (2) 利用荧光共振能量转移 (fluorescence resonance energy transfer, FRET) 效应来实 现, 此类型探针通常由能量供体荧光团、受体苂光 团、连接臂3部分组成. 酶的催化作用使得连接臂断 裂, 供受体苂光团分离, FRET效应抑制, 苂光信号发 生改变(图9) ${ }^{[42,43]}$.

\section{2 研究进展}

早在2006年, Nagano等 ${ }^{[44]}$ 报道了一个基于FRET发 光机理的 $\beta$-半乳糖苷酶标记探针9 (图10). 在未与酶识

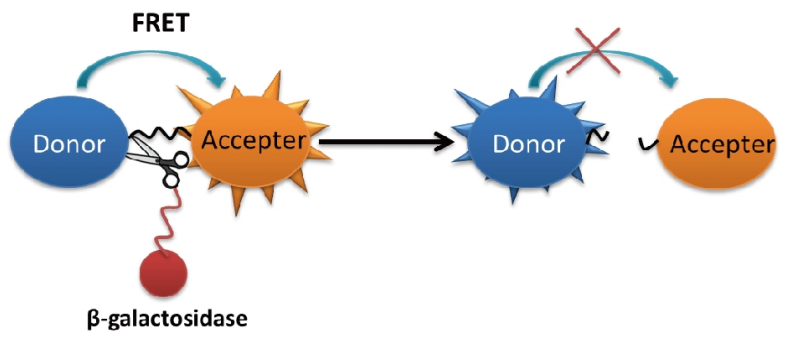

图 9 FRET比率型 $\beta$-半乳糖苷酶荧光探针的设计策略(网 络版彩图)

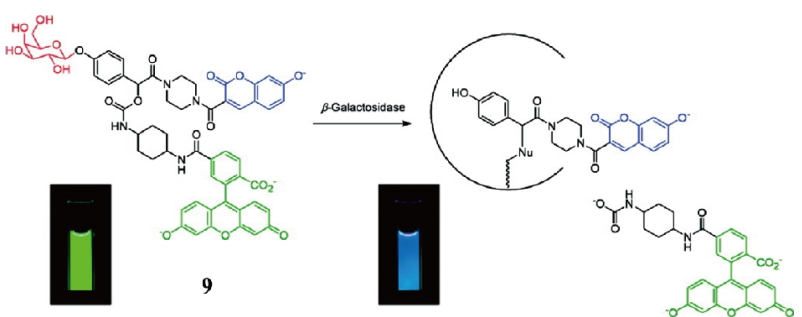

图 10 探针 9 与 $\beta$-半乳糖苷酶的响应机理 ${ }^{[44]}$ (网络版彩图)

别前, 由于荧光共振能量转移, 探针9在 $400 \mathrm{~nm}$ 激发下 并不能观察到 $460 \mathrm{~nm}$ 处香豆素的苂光,但可以得到苂 光素在 $515 \mathrm{~nm}$ 的绿色荧光, 一旦与酶识别, 水解产物 失 去FRET效应, $515 \mathrm{~nm}$ 的绿色荧光逐渐消失, $460 \mathrm{~nm}$ 处的香豆素蓝色苂光逐渐增强, 此苂光共振能量转移 效率可达到 $93 \%$ 以上. 通过 460 和 $515 \mathrm{~nm}$ 的荧光比值 数据可以精确估算 $\beta$-半乳糖苷酶的含量. 该课题组将 此探针应用于LacZ基因转染的HEK293细胞中进行成 像, 并将其应用于蛋白质标记上, 该项研究为未来细 胞中蛋白质的动力学研究提供了研究基础.

2011年, Yang 等 ${ }^{[45]}$ 设计合成了新型的包含 3 个苂 光团以及两个识别位点的探针 $\mathbf{1 0}$ (图11). 该探针采用 PET和FRET协同机制, 不仅可以对 $\beta$-半乳糖苷酶进行 识别, 还可以对磷酸二酯酶进行响应. 不管是在单一 酶还是两种混合酶的作用下,都可以得到高灵敏度的 荧光信号响应. 该探针具有很强的生物稳定性, 因此, 可以广泛应用于细胞成像中. 该探针分子为多功能分 子的设计合成提供了新的思路.

2014年, Kim等 ${ }^{[46]}$ 报道了一个基于ICT效应的新型 双光子比率型苂光探针11 (图12). 该探针以具有优良 双光子发射性能的䒺衍生物作为荧光团, 在其上引入 半乳糖作为识别基团. $\beta$-半乳糖苷酶识别前后, 探针在

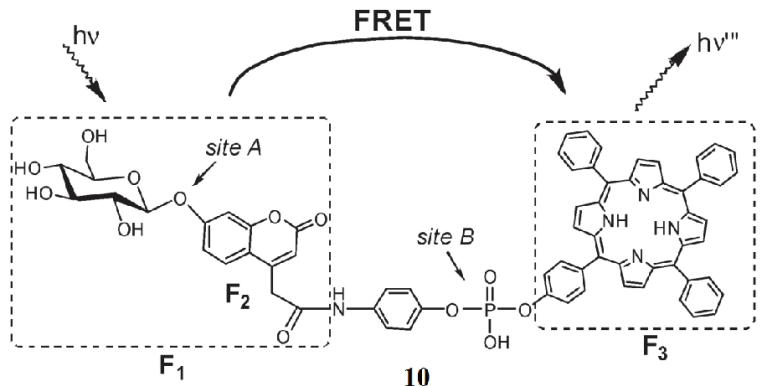

图 11 探针 10 与 $\beta$-半乳糖苷酶的响应机理 ${ }^{[45]}$ 


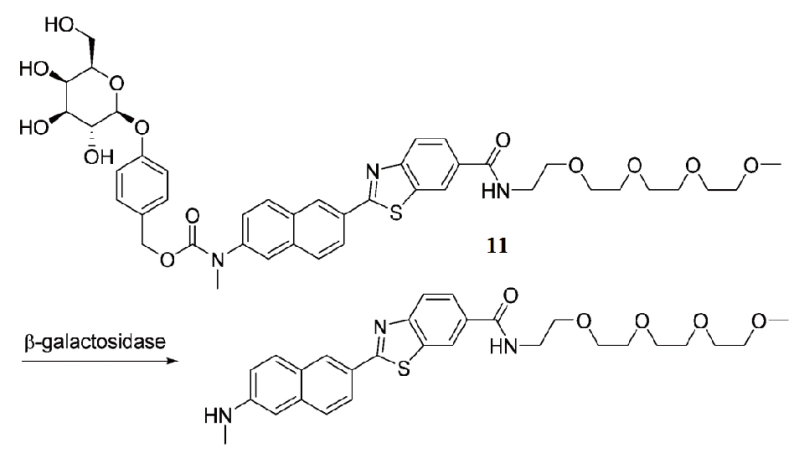

图 12 探针 11 与 $\beta$-半乳糖苷酶的响应机理 ${ }^{[46]}$

461 和 $540 \mathrm{~nm}$ 处出现了显著的荧光强度变化. 利用该 探针成功实现二倍体成纤维细胞(human diploid fibroblasts, HDFs) 以及老鼠表皮组织中 $\beta$-半乳糖苷酶的高分 辨率双光子成像, 进一步验证了细胞衰老状态与 $\beta$-半 乳糖苷酶浓度的关系. 与单光子探针比较, 双光子显 微镜比单光子显微镜具有穿透力强、定位激发以及 延长观察时间等大量优点, 并在检测活细胞组织中的 酶方面具有很好的应用前景.

2016年, Han课题组 ${ }^{[47]}$ 也设计合成了一例基于ICT 效应的双光子比率型荧光探针12 (图 13). 该探针采
用 1,8 -荎酰亚胺为荧光团, 并引入半乳糖作为保护基 团, 使得荧光团的ICT程度发生变化, 在 $365 \mathrm{~nm}$ 光激发 下, 苂光发射峰为 $440 \mathrm{~nm}$. 经 $\beta$-半乳糖苷酶水解作用 后, 探针释放出萗酰亚胺荧光团, 将苂光发射峰红移至 $545 \mathrm{~nm}$, 实现了 $\beta$-半乳糖苷酶的苂光比率响应. 该探针 还被应用于活细胞和活体内 $\beta$-半乳糖苷酶的高分辨率 双光子成像, 体现了该探针在生物医学中的应用价值.

2016年, 本课题组 ${ }^{[48]}$ 报道了一个以苯并吡喃腈为 母体的近红外苂光探针 13 (图14). 该探针将苯并吡喃

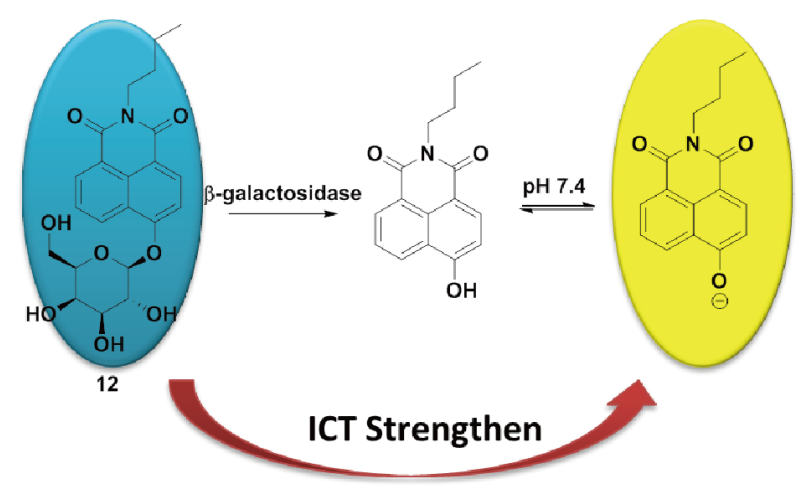

图 13 探针 12 与 $\beta$-半乳糖苷酶的响应机理 ${ }^{[4]}$ (网络版彩图)

(a)
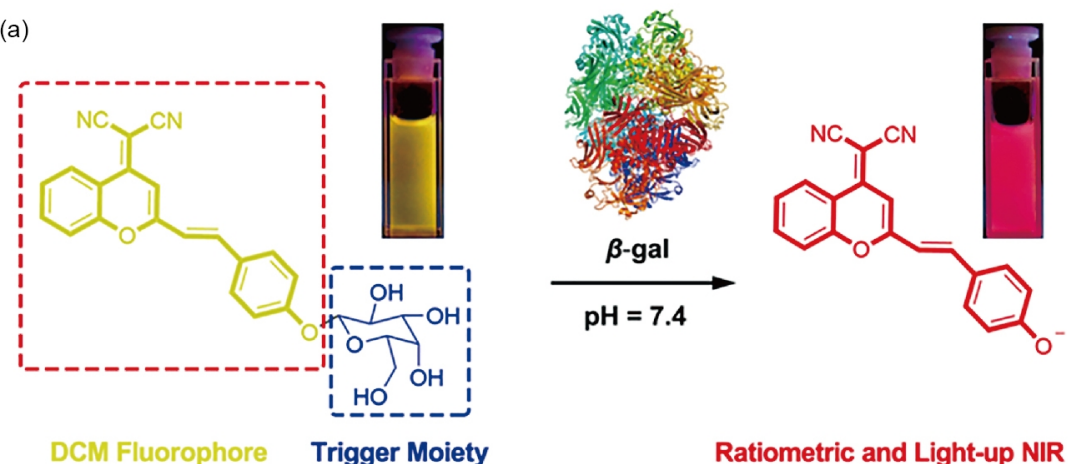

Ratiometric and Light-up NIR Mode

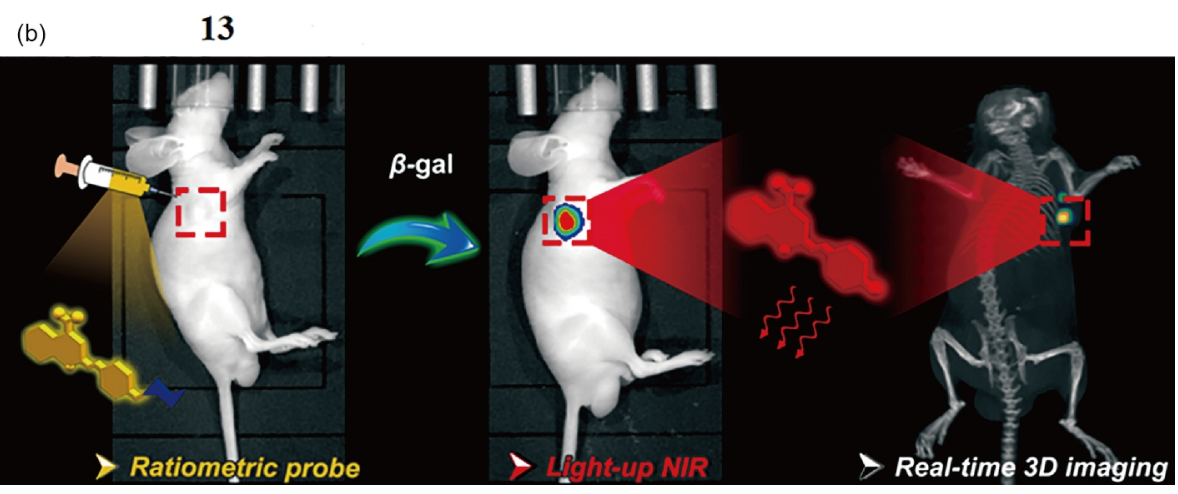

图 14 探针 13 的响应机理(a)及小鼠体内成像图(b) ${ }^{[48]}$ (网络版彩图) 
腈的衍生物上的酚羟基用半乳糖保护起来, 同样采用 ICT 发光机理, 在与 $\beta$-半乳糖苷酶水解作用后, 荧光最 大发射波长从 $500 \mathrm{~nm}$ 红移至 $685 \mathrm{~nm}$, 实现了对 $\beta$-半乳 糖苷酶的比率型苂光响应. 基于探针 13 良好的荧光性 能以及探针的比率响应效果, 被成功应用于卵巢癌细 胞内苂光成像, 并且在结肠癌小鼠模型内实现了对 $\beta$ 半乳糖苷酶的实时在体、原位检测, 并获取了高分辨 三维活体成像信号, 实现对肿瘤组织的精确定位. 该 工作进一步被Nature Methods进行了亮点报道 ${ }^{[9]}$, 并指 出, $\beta$-半乳糖苷酶是一个重要的肿瘤标记物, 故其在体 内的成像研究十分重要, 为体内酶活性的实时跟踪监 测的临床应用奠定了良好的研究基础.

\section{4 结论}

针对 $\beta$-半乳糖苷酶的检测识别, 本文主要综述了 反应激活型荧光探针的设计策略以及研究进展. 目前 关于 $\beta$-半乳糖苷酶的苂光探针在苂光增强型以及比率 型探针的研究中取得了一定的进展, 但仍有一些问题 需要解决 ${ }^{[00,51]}$ : (1) 目前新型靶向识别不同细胞器中酶 的苂光探针的报道还很少见, 而不同细胞器中酶活性 的不同可能会引起不同的疾病, 因此这会成为广大科 研工作者的研究热点; (2) 设计合成水溶性良好, 并且 能与生物体系相容; (3) 对活体内深层组织中 $\beta$-半乳糖 苷酶活性內源性检测、代谢过程和原位成像的近红 外苂光探针的研究仍是一个重要的领域.

\section{参考文献}

1 Lu P, Gao M, Cai X, Zhang R, Li K, Feng G, Tong A, Liu B. J Mater Chem B, 2015, 3: 9168-9172

Wijdeven RH, Neefjes J, Ovaa H. Trends Cell Biol, 2014, 24: 751-760

Geng YQ, Guan JT, Xu XH, Fu YC. Biochem Biophys Res Commun, 2010, 396: 866-869

4 Sakabe M, Asanuma D, Kamiya M, Iwatate RJ, Hanaoka K, Terai T, Nagano T, Urano Y. J Am Chem Soc, 2013, 135: 409-414

5 Sosińska P, Mikuła-Pietrasik J, Ryżek M, Naumowicz E, Książek K. Biogerontology, 2014, 15: 407-413

6 Yu JX, Kodibagkar VD, Hallac RR, Liu L, Mason RP. Bioconjugate Chem, 2012, 23: 596-603

7 Geiger B, Nguyen HM, Wenig S, Nguyen HA, Lorenz C, Kittl R, Mathiesen G, Eijsink VGH, Haltrich D, Nguyen TH. Biochem Eng J, 2016, 116: $45-53$

8 Wang J, Wu L, Ren J, Qu X. Small, 2012, 8: 166-166

9 Chikkaveeraiah BV, Bhirde AA, Morgan NY, Eden HS, Chen X. ACS Nano, 2012, 6: 6546-6561

10 Zhou Y, Yin H, Li X, Li Z, Ai S, Lin H. Biosens Bioelectron, 2016, 86: 508-515

11 Du J, Zhu B, Peng X, Chen X. Small, 2014, 10: 3461-3479

12 Zhou J, Liu Q, Feng W, Sun Y, Li F. Chem Rev, 2015, 115: 395-465

13 Ding L, Bai Y, Cao Y, Ren G, Blanchard GJ, Fang Y. Langmuir, 2014, 30: 7645-7653

14 Na SY, Kim HJ. Dyes Pigments, 2016, 134: 526-530

15 Zhang C, Wang D, Zhang L, Guo JF, Ren AM. RSC Adv, 2016, 6: 70960-70971

16 Wu X, Zhu W. Chem Soc Rev, 2015, 44: 4179-4184

17 Shao A, Guo Z, Zhu S, Zhu S, Shi P, Tian H, Zhu W. Chem Sci, 2014, 5: 1383-1389

18 Li X, Wu Y, Liu Y, Zou X, Yao L, Li F, Feng W. Nanoscale, 2014, 6: 1020-1028

19 Hong G, Antaris AL, Dai H. Nat Biomed Eng, 2017, 1: 0010

20 Oushiki D, Kojima H, Takahashi Y, Komatsu T, Terai T, Hanaoka K, Nishikawa M, Takakura Y, Nagano T. Anal Chem, 2012, 84: 4404-4410

21 Broome AM, Ramamurthy G, Lavik K, Liggett A, Kinstlinger I, Basilion J. Bioconjugate Chem, 2015, 26: 660-668

22 Liu L, Mason RP. PLoS One, 2010, 5: e12024

23 Karton-Lifshin N, Vogel U, Sella E, Seeberger PH, Shabat D, Lepenies B. Org Biomol Chem, 2013, 11: 2903-2910

24 Kim EJ, Kumar R, Sharma A, Yoon B, Kim HM, Lee H, Hong KS, Kim JS. Biomaterials, 2017, 122: 83-90

25 Qian L, Li L, Yao SQ. Acc Chem Res, 2016, 49: 626-634

26 Edgington LE, Verdoes M, Bogyo M. Curr Opin Chem Biol, 2011, 15: 798-805

27 Ichikawa Y, Kamiya M, Obata F, Miura M, Terai T, Komatsu T, Ueno T, Hanaoka K, Nagano T, Urano Y. Angew Chem Int Ed, 2014, 53: $6772-6775$

Sicard C, Shek N, White D, Bowers RJ, Brown RS, Brennan JD. Anal Bioanal Chem, 2014, 406: 5395-5403

Mandal PK, Cattiaux L, Bensimon D, Mallet JM. Carbohyd Res, 2012, 358: 40-46 
Urano Y, Kamiya M, Kanda K, Ueno T, Hirose K, Nagano T. J Am Chem Soc, 2005, 127: 4888-4894

Kamiya M, Kobayashi H, Hama Y, Koyama Y, Bernardo M, Nagano T, Choyke PL, Urano Y. J Am Chem Soc, 2007, 129: 3918-3929

Kamiya M, Asanuma D, Kuranaga E, Takeishi A, Sakabe M, Miura M, Nagano T, Urano Y. J Am Chem Soc, 2011, 133: 12960-12963

Egawa T, Koide Y, Hanaoka K, Komatsu T, Terai T, Nagano T. Chem Commun, 2011, 47: 4162-4164

Asanuma D, Sakabe M, Kamiya M, Yamamoto K, Hiratake J, Ogawa M, Kosaka N, Choyke PL, Nagano T, Kobayashi H, Urano Y. Nat Commun, 2015, 6: 6463

Koide Y, Urano Y, Yatsushige A, Hanaoka K, Terai T, Nagano T. J Am Chem Soc, 2009, 131: 6058-6059

Han J, Han MS, Tung CH. Mol BioSyst, 2013, 9: 3001-3008

Redy-Keisar O, Kisin-Finfer E, Ferber S, Satchi-Fainaro R, Shabat D. Nat Protoc, 2013, 9: 27-36

Wang X, Sun J, Zhang W, Ma X, Lv J, Tang B. Chem Sci, 2013, 4: 2551-2556

Santra M, Roy B, Ahn KH. Org Lett, 2011, 13: 3422-3425

Chen X, Wang J, Cui J, Xu Z, Peng X. Tetrahedron, 2011, 67: 4869-4873

Yu F, Li P, Song P, Wang B, Zhao J, Han K. Chem Commun, 2012, 48: 2852-2854

Zhang X, Xiao Y, Qian X. Angew Chem Int Ed, 2008, 47: 8025-8029

Yuan L, Lin W, Zheng K, Zhu S. Acc Chem Res, 2013, 46: 1462-1473

Komatsu T, Kikuchi K, Takakusa H, Hanaoka K, Ueno T, Kamiya M, Urano Y, Nagano T. J Am Chem Soc, 2006, 128: 15946-15947

Li Y, Wang H, Li J, Zheng J, Xu X, Yang R. Anal Chem, 2011, 83: 1268-1274

Lee HW, Heo CH, Sen D, Byun HO, Kwak IH, Yoon G, Kim HM. Anal Chem, 2014, 86: 10001-10005

Zhang XX, Wu H, Li P, Qu ZJ, Tan MQ, Han KL. Chem Commun, 2016, 52: 8283-8286

Gu K, Xu Y, Li H, Guo Z, Zhu S, Zhu S, Shi P, James TD, Tian H, Zhu WH. J Am Chem Soc, 2016, 138: 5334-5340

Tracking $\beta$-galactosidase activity in vivo. Nat Methods, 2016, 13: 470

Zhu W. Sci China Chem, 2016, 59: 203-204

Wu X, Shao A, Zhu S, Guo Z, Zhu W. Sci China Chem, 2016, 59: 62-69

\title{
Progress in fluorescent sensors for $\beta$-galactosidase
}

\author{
Meng $\mathrm{Li}^{1}$, Tianchu $\mathrm{Gao}^{2}$, Weihong $\mathrm{Zhu}^{3 *}$ \\ ${ }^{1}$ Department of Environmental Science and Engineering, North China Electric Power University, Baoding 071000, China \\ ${ }^{2}$ First Central Hospital of Baoding, Baoding 071000, China \\ ${ }^{3}$ Key Laboratory for Advanced Materials Ministry of Education and Institute of Fine Chemicals, Shanghai Key Laboratory of Functional Materials \\ Chemistry, School of Chemistry and Molecular Engineering, East China University of Science and Technology, Shanghai 200237, China \\ *Corresponding author (email: whzhu@ecust.edu.cn)
}

\begin{abstract}
: $\beta$-galactosidase, a hydrolytic enzyme in cell lysosomes, plays a crucial role in catalytic hydrolysis of glycosidic bond with the lactose into galactose. In addition, it is also an important indication for $\beta$-galactosidase defect disease diagnosis and basic research in the field of genetics. Therefore, $\beta$-galactosidase detection is of great significance in the area of life sciences and medicine. In recent years, fluorescent probe for $\beta$-galactosidase recognition has drawn excessive attention due to its good selectivity, high sensitivity and high spatiotemporal resolution imaging. Many fluorescent probes have been developed for $\beta$-galactosidase visual imaging in biological systems. In this paper, the design strategy and research progress of $\beta$-galactosidase fluorescent probes are reviewed, and the future research direction is discussed.
\end{abstract}

Keywords: $\beta$-galactosidase, fluorescence, probe, bioimaging

doi: $10.1360 / \mathrm{N} 032017-00030$ 